\title{
Supra-annular sizing for transcatheter valve implantation in bicuspid aortic stenosis
}

\author{
Raban Jeger ${ }^{1}$, Oliver Reuthebuch ${ }^{1}$, Gregor Fahrni ${ }^{1}$, Jean-Claude Laborde ${ }^{2}$, Rolf Vogel ${ }^{3}$, Christoph Kaiser ${ }^{1}$ \\ ${ }^{1}$ Department of Cardiology and Heart Surgery, University Hospital Basel, Basel, Switzerland \\ ${ }^{2}$ Department of Cardiology, St. George's University Hospitals NHS Foundation Trust, London, UK \\ ${ }^{3}$ Department of Cardiology, Bürgerspital Solothurn, Solothurn, Switzerland
}

Adv Interv Cardiol 2018; 14, 2 (52): 187-190

DOI: https://doi.org/10.5114/aic.2018.76411

\section{Introduction}

Transcatheter aortic valve implantation (TAVI) is an established treatment option for symptomatic severe aortic stenosis in high [1] and intermediate risk patients [2]. With a prevalence of $1 \%$ to $2 \%$ in countries outside China, bicuspid aortic valve (BAV) disease is a common congenital malformation [3] that often results in premature aortic stenosis. Since BAV represents a challenge for interventional treatment [4], it traditionally has been considered as a relative contraindication against TAVI. The Lotus valve (Boston Scientific Corp, Marlborough $M A)$ is a mechanically expandable, fully repositionable and retrievable second-generation TAVI prosthesis with an outer adaptive seal that facilitates accurate positioning, early valve function, and hemodynamic stability during deployment, and minimizes paravalvular leakage [5]. Therefore, it might be a good treatment option for BAV stenosis.

For planning TAVI procedures, an in-depth analysis of multidetector computed tomography (MDCT) images is crucial. In most reports of interventional treatment of BAV stenosis, preprocedural measurements were done according to current guidelines [6]. However, such measurements might lead to inaccurate annular sizing and prosthesis selection in BAV disease. Specifically, measurement of the basal ring may lead to the selection of too large prostheses, potentially leading to suboptimal results, or may make the intervention impossible due to non-availability of the necessary large valve sizes.

\section{Aim}

Therefore, an alternative process of measurement may be preferred. We therefore aimed to use a new algorithm for MDCT analysis, i.e., supra-annular measure- ment of the valve opening area at the level of the maximal calcification, to assess the correct aortic valve area opening, and compared it to the conventional analysis algorithm in terms of aortic valve area and diameters in a series of patients with severe BAV stenosis undergoing TAVI using the mechanically expandable Lotus valve.

\section{Material and methods}

Between January 2015 and February 2017, patients with severe symptomatic aortic stenosis undergoing TAVI at the University Hospital Basel, Switzerland, were screened for the presence of BAV. Before the intervention, patients underwent transthoracic echocardiography, coronary angiography, and MDCT, and were reviewed by the institutional heart team to determine the best treatment approach. Bicuspid aortic valve patients underwent TAVI using Lotus valves via the transfemoral or transsubclavian route, as indicated. Data were collected prospectively, and all patients signed informed consent to be followed within a registry. The study was approved by the responsible ethics committee.

The preprocedural MDCT was both ECG-gated and contrast-enhanced and acquired following established institutional scan protocols. All MDCT datasets were evaluated by a single experienced reader for quantitative measurements using the 3 mensio software (Pie Medical Imaging BV, Maastricht, The Netherlands) and by two additional readers to define valve morphology. Valve morphology was classified according to the MDCT data [7]. Quantitative analysis included measurements of annular dimensions by means of planimetry using maximal and minimal diameters and perimeter in systole, and was performed according to current guidelines [6]. In addition to the conventional annular measurement, supra-annular

\section{Corresponding author:}

Prof. Raban Jeger, Cardiology and Heart Surgery, University Hospital Basel, Petersgraben 4, 4031 Basel, Switzerland, phone: +41 612652525, e-mail: raban.jeger@usb.ch

Received: 20.01.2018, accepted: 25.02.2018. 
Table I. Baseline characteristics

\begin{tabular}{|c|c|}
\hline Parameter & Value \\
\hline \multicolumn{2}{|l|}{ Demographic data: } \\
\hline Age [years] & $78.75 \pm 4.92$ \\
\hline Sex female (\%) & 38 \\
\hline EuroSCORE II (\%) & $3.05 \pm 2.01$ \\
\hline \multicolumn{2}{|l|}{ Patients' history } \\
\hline Arterial hypertension (\%) & 88 \\
\hline Hyperlipidemia (\%) & 50 \\
\hline Diabetes mellitus (\%) & 38 \\
\hline Family history (\%) & 0 \\
\hline Current smoking (\%) & 63 \\
\hline Prior myocardial infarction (\%) & 13 \\
\hline Prior coronary artery bypass graft surgery (\%) & 13 \\
\hline Prior stroke (\%) & 0 \\
\hline Renal failure (\%) & 13 \\
\hline Atrial fibrillation (\%) & 50 \\
\hline \multicolumn{2}{|l|}{ Echocardiographic data: } \\
\hline Left ventricular ejection fraction (\%) & $58.0 \pm 7.82$ \\
\hline Maximal aortic gradient [mm Hg] & $82.0 \pm 19.2$ \\
\hline Mean aortic gradient [mm Hg] & $54.43 \pm 12.75$ \\
\hline Aortic valve area $\left[\mathrm{cm}^{2}\right]$ & $0.83 \pm 0.27$ \\
\hline \multicolumn{2}{|l|}{ Clinical data: } \\
\hline \multicolumn{2}{|l|}{ Dyspnea (\%): } \\
\hline I & 0 \\
\hline 11 & 25 \\
\hline III & 62.5 \\
\hline IV & 12.5 \\
\hline \multicolumn{2}{|l|}{ Angina pectoris (\%): } \\
\hline 1 & 0 \\
\hline 2 & 37.5 \\
\hline 3 & 0 \\
\hline 4 & 0 \\
\hline \multicolumn{2}{|l|}{ Multidetector CT data: } \\
\hline \multicolumn{2}{|l|}{ Bicuspid aortic valve type (\%): } \\
\hline BAV-AP type 1 & 37.5 \\
\hline BAV-AP type 2 & 0 \\
\hline BAV-RL type 3 & 50 \\
\hline BAV-RL type 4 & 12.5 \\
\hline BAV-RL type 5 & 0 \\
\hline
\end{tabular}

Continuous variables are presented as mean \pm standard deviation. Categorical variables are presented as percentages. measurement was done measuring the maximal length of the valve opening (intercommissural length) and a second diameter perpendicular to this maximal diameter at the level of the maximal leaflet calcification, again measured in systole. Effective valve size was chosen according to the supra-annular measurement.

Follow-up including clinical assessment and transthoracic echocardiography was done after 30 days. Study endpoints included the Valve Academic Research Consortium-2 criteria [8] and echocardiographic data such as aortic valve gradient, degree of aortic regurgitation, and left ventricular ejection fraction. Postprocedural aortic regurgitation was defined as the sum of para- and transvalvular regurgitation, while the severity was graded as none or trivial, mild, moderate, or severe by means of qualitative assessment.

\section{Statistical analysis}

Statistical analyses were done using the GraphPad software (GraphPad Software Inc, La Jolla CA). Continuous variables are presented as mean \pm standard deviation and compared with Student's $t$ test. Categorical variables are presented as percentages. All hypothesis testing was 2-tailed, and a $p$-value of $<0.05$ was considered statistically significant.

\section{Results}

\section{Baseline characteristics}

From 253 patients undergoing TAVI, 108 (42.7\%) were treated with an Edwards Sapien 3, 73 (28.9\%) with a Lotus, 47 (18.5\%) with a Portico, 11 (4.3\%) with a CoreValve Evolut R, 8 (3.2\%) with a JenaValve, 5 (2\%) with a Direct Flow, and $1(0.4 \%)$ with an NVT valve. Overall, there were 8 (3.2\%) patients with BAV, who were treated with a Lotus. These patients had a mean age of $78.8 \pm 4.9$ years with a logistic EuroSCORE II of $3.1 \pm 2.0 \%$ and suffered from either dyspnea or angina pectoris. Mean aortic gradient was $51.43 \pm 12.75 \mathrm{~mm} \mathrm{Hg}$ with an aortic valve area of $0.83 \pm 0.27 \mathrm{~cm}^{2}$. Anatomy of the valve was in $50 \%$ BAVRL type 3, in 37.5\% BAV-AP type 1, and in $12.5 \%$ BAV-RL type 4 [7]. The baseline demographic, echocardiographic, and MDCT data are summarized in Table I.

\section{Multidetector computed tomography measurements}

Supra-annular measurement resulted in both smaller valve area (436.54 $\pm 72.83 \mathrm{~mm}^{2}$ vs. $536.73 \pm 88.33 \mathrm{~mm}^{2}$, $p=0.0267)$ and mean diameter $(23.49 \pm 1.98 \mathrm{~mm}$ vs. $26.06 \pm 2.18 \mathrm{~mm}, p=0.0271)$ compared with annular measurement, which resulted in the selection of smaller valve sizes or made the use of the Lotus valve possible at all, respectively (Figure 1). While Lotus valve implantation was not possible with conventional measurement in $37.5 \%$ of cases, supra-annular measurement revealed a sufficient annular size in all patients. 


\section{Outcome}

Patients underwent TAVI in $87.5 \%$ of cases via the transfemoral and in $12.5 \%$ of cases via the transsubclavian route. Procedural success was $100 \%$. The implantation of a Lotus valve resulted in a decrease of both peak and mean gradient to $26.25 \pm 5.12$ and $14.43 \pm 6.58 \mathrm{~mm} \mathrm{Hg}$ at 30 days, respectively (both $p<0.0001$ ). Regarding clinical endpoints at 30 days, 2 patients underwent permanent pacemaker implantation due a high-degree AV block (25\%) and one patient suffered from a valve thrombosis (12.5\%), while no patients suffered from either death or stroke. There was no aortic regurgitation at follow-up.

\section{Discussion}

Due to its specific anatomic characteristics, BAV stenosis is a challenge for interventional treatment [4]. Registry data show that TAVI in BAV stenosis compared with tricuspid aortic valve stenosis is associated with lower rates of procedural success, higher rates of pacemaker implantation, and more paravalvular leakage; however, long-term mortality was similar [9-11]. While first-generation TAVI prostheses were associated with higher rates of aortic root injury and paravalvular leakage, second-generation prostheses showed similar, favorable results for tricuspid and bicuspid aortic valves, with low rates of annular rupture, relevant aortic regurgitation, or second valve implantation [10, 12].

Based on its mechanical properties, the Lotus valve might be ideal to prevent aortic root injury and regurgitation, specifically in high-risk patients with BAV stenosis. Accordingly, early reports of interventional treatment of BAV stenosis with the Lotus valve showed a good clinical and hemodynamic result without relevant paravalvular leakage $[13,14]$. The Lotus valve is available in three sizes, i.e., $23 \mathrm{~mm}, 25 \mathrm{~mm}$, and $27 \mathrm{~mm}$. Therefore, it can be used for diameters of up to $27 \mathrm{~mm}$ only, which limits the use in large aortic annuli. Currently, the use of the Lotus valve has been suspended due to technical issues, but its return to the market is expected soon. Bicuspid aortic valve is characterized by the presence of only two functional cusps and an oval orifice, and can be classified by different systems $[7,15]$. The number of detected raphes can be used to group BAV into the 3 following types: type 0 (no raphe); type 1 (1 raphe); and, type 2 (2 raphes) [15]. Other classification systems use imaging modalities such as MDCT for phenotypic classification that has been used in the current analysis. In this classification system, two main types are defined, i.e., fusion of the right and left coronary cusp (type BAV-AP) and fusion of the right or left coronary cusp and the non-coronary cusp (BAV-RL) with a subdivision in types 1 and 2 in BAV-AP and 3 to 5 in BAV-RL [7].

Current guidelines for preprocedural MDCT require that annular sizing and prosthesis selection are based on a basal ring that is measured at the level immediately below the 3 lowest insertion points of the aortic cusps in sys-

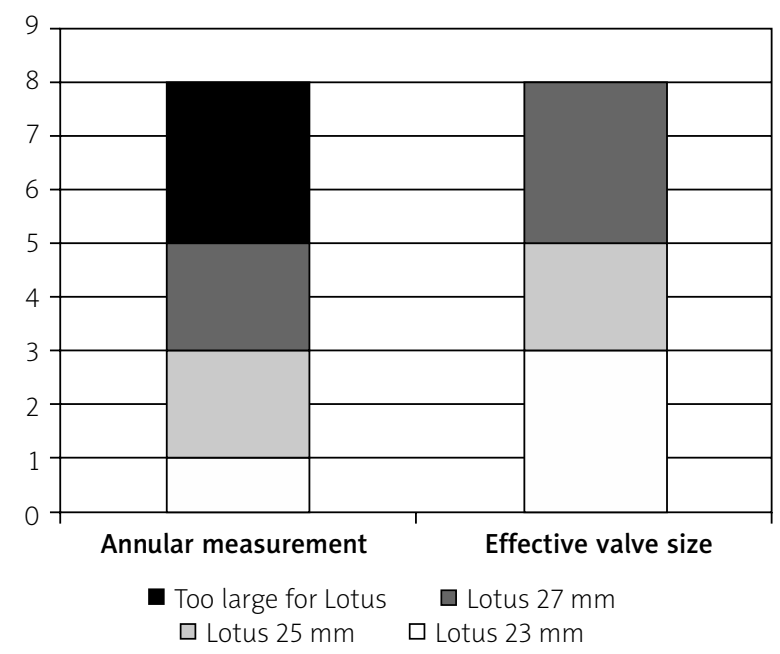

Figure 1. Valve size selection according to annular and supra-annular measurements

Black - too large for Lotus, dark gray - Lotus $27 \mathrm{~mm}$, light gray - Lotus $25 \mathrm{~mm}$, white - Lotus $23 \mathrm{~mm}$.

tole [6]. In the current analysis, an additional supra-annular measurement of the valve opening area at the level of maximal leaflet calcification was performed and showed a considerable lower valve area, which resulted in the choice of smaller valves and allowed the Lotus valve to be used in all cases, which was not possible with conventional measurement only. Of note, the unique properties of repositionability and retrievability combined with the leak tightness of the Lotus valve made it possible to choose smaller valves without risk of embolization and aortic regurgitation, which resulted in favorable clinical outcomes.

This analysis has some limitations. In the present study, we did not compare tricuspid vs. bicuspid valves from our database. Therefore, there is no formal comparison between the two anatomical groups. In addition, the small number of patients in the present case series does not allow the results to be generalized to a larger number of patients and precludes definitive conclusions regarding the comparison of the two MDCT measurement algorithms regarding outcome.

\section{Conclusions}

Bicuspid aortic valve stenosis may be treated with good success with TAVI in a wide array of patients if MDCT measurements are performed accurately. Supra-annular measurements in combination with conventional annular measurements might optimize the pre-interventional evaluation of the patients, and second generation TAVI prostheses such as the Lotus valve might be a good solution to accommodate BAV anatomy.

\section{Acknowledgments}

Funding - Cardiology, University Hospital Basel, Switzerland. 


\section{Conflict of interest}

Raban Jeger and Jean-Claude Laborde are proctors for Boston Scientific. Other authors declare no conflict of interest.

\section{References}

1. Smith CR, Leon MB, Mack MJ, et al. Transcatheter versus surgical aortic-valve replacement in high-risk patients. N Engl J Med 2011; 364: 2187-98.

2. Leon MB, Smith CR, Mack MJ, et al.; Investigators P. Transcatheter or surgical aortic-valve replacement in intermediate-risk patients. N Engl J Med 2016; 374: 1609-20.

3. Michelena HI, Prakash SK, Della Corte A, et al.; Investigators B. Bicuspid aortic valve: identifying knowledge gaps and rising to the challenge from the International Bicuspid Aortic Valve Consortium (BAVCon). Circulation 2014; 129: 2691-704.

4. Mylotte D, Lefevre T, Søndergaard L, et al. Transcatheter aortic valve replacement in bicuspid aortic valve disease. J Am Coll Cardiol 2014; 64: 2330-9.

5. Meredith IT, Walters DL, Dumonteil N, et al. 1-year outcomes with the fully repositionable and retrievable Lotus transcatheter aortic replacement valve in 120 high-risk surgical patients with severe aortic stenosis: results of the REPRISE II Study. JACC Cardiovasc Interv 2016; 9: 376-84.

6. Achenbach S, Delgado V, Hausleiter J, et al. SCCT expert consensus document on computed tomography imaging before transcatheter aortic valve implantation (TAVI)/transcatheter aortic valve replacement (TAVR). J Cardiovasc Comput Tomogr 2012; 6: 366-80.

7. Kang JW, Song HG, Yang DH, et al. Association between bicuspid aortic valve phenotype and patterns of valvular dysfunction and bicuspid aortopathy: comprehensive evaluation using MDCT and echocardiography. JACC Cardiovasc Imaging 2013; 6: 150-61.

8. Kappetein AP, Head SJ, Généreux P, et al.; Consortium-2 VAR. Updated standardized endpoint definitions for transcatheter aortic valve implantation: the Valve Academic Research Consortium-2 consensus document. Eurolntervention 2012; 8: 782-95.

9. Bauer T, Linke A, Sievert H, et al. Comparison of the effectiveness of transcatheter aortic valve implantation in patients with stenotic bicuspid versus tricuspid aortic valves (from the German TAVI Registry). Am J Cardiol 2014; 113: 518-21.

10. Yoon SH, Bleiziffer S, De Backer O, et al. Procedural and clinical outcomes in transcatheter aortic valve replacement for bicuspid versus tricuspid aortic valve stenosis. J Am Coll Cardiol 2017; 69: 2579-89.

11. Yousef A, Simard T, Pourdjabbar A, et al. Performance of transcatheter aortic valve implantation in patients with bicuspid aortic valve: systematic review. Int J Cardiol 2014; 176: 562-4.

12. Yoon SH, Lefèvre T, Ahn JM, et al. Transcatheter aortic valve replacement with early- and new-generation devices in bicuspid aortic valve stenosis. J Am Coll Cardiol 2016; 68: 1195-205.

13. Seeger J, Gonska B, Rodewald C, et al. Bicuspid aortic stenosis treated with the repositionable and retrievable Lotus valve. Can J Cardiol 2016; 32: 135.e17-9.

14. Chan AW, Wong D, Charania J. Transcatheter aortic valve replacement in bicuspid aortic stenosis using Lotus Valve System. Catheter Cardiovasc Interv 2017; 90: 157-63.
15. Sievers HH, Schmidtke C. A classification system for the bicuspid aortic valve from 304 surgical specimens. J Thorac Cardiovasc Surg 2007; 133: 1226-33. 